\title{
Multi-Fidelity Aerodynamic Optimization of a Helicopter Rotor Blade
}

\author{
Joëlle Bailly $^{*}$, and Didier Bailly. ${ }^{\dagger}$ \\ ONERA, The French Aerospace Lab, Meudon, 92190, FRANCE
}

\footnotetext{
Research Engineer, Aerodynamics Aeroelastics and Acoustics Department, Helicopters, Propellers and Turbomachinery Unit, 8, rue des Vertugadins, 92190 Meudon, joelle.zibi@onera.fr.

† Research Engineer, Aerodynamics Aerelastics and Acoustics Department, Civil Aircraft Unit, 8, rue des Vertugadins, 92190 Meudon, didier.bailly@onera.fr
} 
A multi-fidelity optimization technique is applied to the design of a helicopter rotor blade to improve its performance in forward flight. This optimization technique is based on surrogate model that replace the high-fidelity CFD/CSD simulations necessary to capture the three-dimensional unsteady effects generated in the flow field of a complex blade geometry. The single low-fidelity model based on Kriging methodology and generated by lifting-line simulations, leads to a power benefit of $2.5 \%$, which is not reproducible by an $a$ posteriori high-fidelity CSD/CFD computation. The optimization procedure using CoKriging surrogate models based on two levels of fidelity (lifting line and CSD/CFD simulations) leads to a realistic blade planform, for which the power benefit is estimated at 2.2\%. This optimized solution, obtained after a factor 6 reduction in CPU time, shows the advantages of using a Co-Kriging surrogate model (rather than a single-fidelity Kriging model) for aerodynamic optimizations.

\section{Nomenclature}

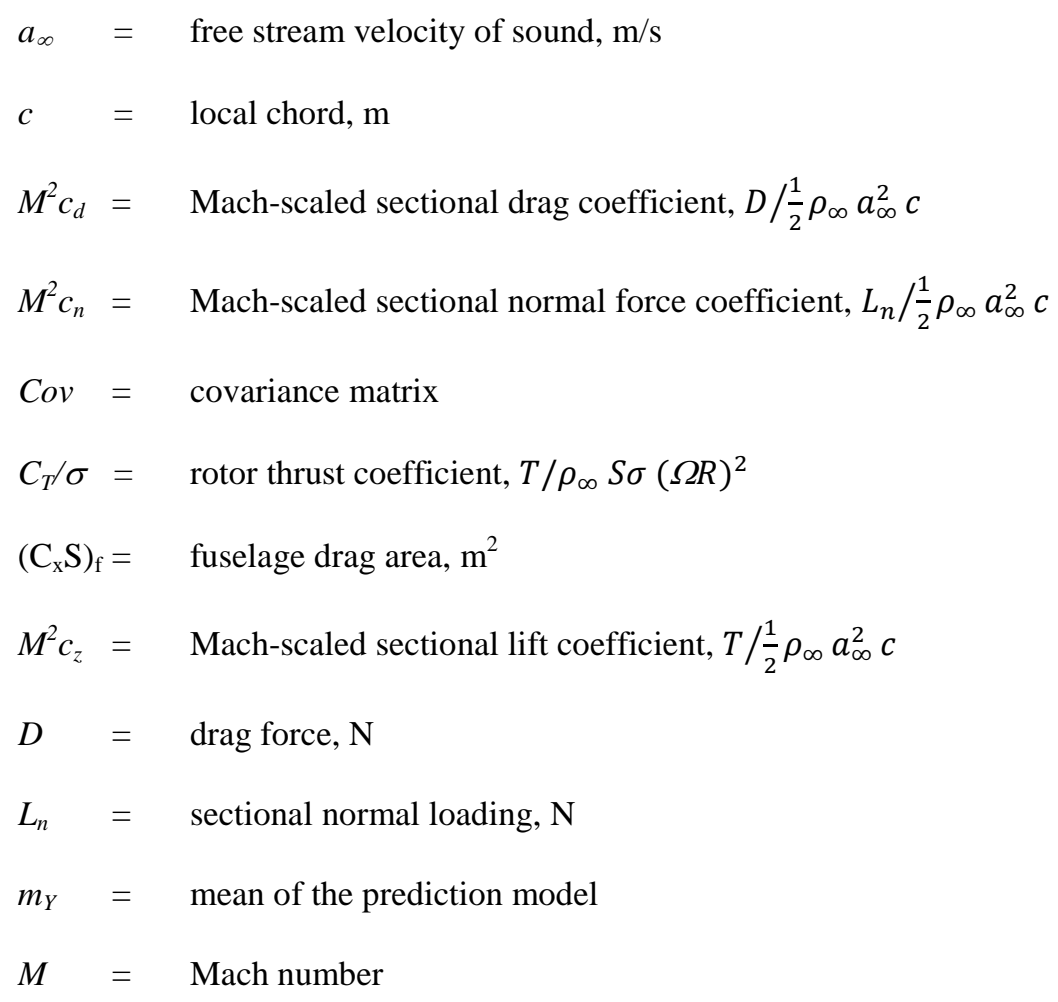




$$
\begin{aligned}
& \mathrm{M}_{\mathrm{x}}=\text { rotor hub rolling moment, } \mathrm{Nm} \\
& N_{b}=\text { number of rotor blades } \\
& P \quad=\quad \text { required rotor power, } \mathrm{kW} \\
& P_{\text {ind }}=\text { induced power, } \mathrm{kW} \\
& r \quad=\quad \text { radial coordinate, } \mathrm{m} \\
& R \quad=\quad \text { rotor radius, } \mathrm{m} \\
& \boldsymbol{R}_{i j}=\text { correlation between two data points, } \mathrm{i} \text { and } \mathrm{j} \\
& S=\text { rotor disk surface, } \mathrm{m}^{2}, \pi R^{2} \\
& T=\text { rotor thrust, } \mathrm{N} \\
& V_{h}=\text { advancing velocity, } \mathrm{kt} \\
& \boldsymbol{X}=\quad \text { vector of independent variables } \\
& \boldsymbol{Y}=\text { output of the computational functions } \\
& \rho_{\infty}=\text { free stream air density, } \mathrm{kg} / \mathrm{m}^{3} \\
& \sigma=\mathrm{N}_{\mathrm{b}} \mathrm{C} / \pi \mathrm{R} \text {, rotor solidity } \\
& \sigma^{2}=\quad \text { Gaussian model variance } \\
& \Omega=\text { rotor rotational speed, } \mathrm{rad} / \mathrm{s} \\
& \theta_{0} \quad=\quad \text { collective pitch angle, deg } \\
& \theta_{1 c}=\text { lateral cyclic pitch angle, deg } \\
& \theta_{1 s}=\text { longitudinal cyclic pitch angle, deg } \\
& \theta_{e l}=\text { longitudinal cyclic pitch angle, deg } \\
& \psi \quad=\quad \text { azimuth angle, deg } \\
& \phi=\text { probability density function } \\
& \Phi=\text { cumulative distributive function }
\end{aligned}
$$

$c, d, e=$ cheap, difference and expensive functions 


\section{Introduction}

THE aerodynamic optimization of helicopter rotor blades is a complex and challenging problem due to unsteady flow phenomena. For instance, in forward flight, transonic effects on the advancing side of the blade and dynamic stall on the retreating side can be encountered. Furthermore, the effects between the aerodynamic behavior and the elastic response of an optimized rotor blade are considered through a fluid-structure coupling at a great computational cost. Historically, aerodynamic optimization procedures were based on the coupling between low-fidelity and fast computational codes, generally based on the lifting line theory, and an optimizer. Two types of optimizer were typically applied, either gradient-based (limited number of evaluations, but with the risk of reaching a local minimum of the objective function), or based on genetic algorithms (which require a great number of evaluations, over a large research domain, which improves the capability to reach the global minimum of the objective function).

Single-objective optimizations with gradient-descent are among the first approaches to have been used due to their efficiency and rapidity. At NASA, Walsh and Bingham assessed the power minimization problem sequentially, first optimizing in hover flight, and then handling the constraints in forward flight. The chosen optimizer was the CONMIN algorithm [1]. At ONERA, the CONMIN optimizer has been coupled with the R85 comprehensive code [2] to optimize the geometry (chord, twist, sweep, anhedral) of a rotor blade to reduce its required power in forward flight while constraining the values of the pitch link loads [3].

Recently, the use of gradient-based algorithms formulated by the discrete steady adjoint of the RANS equations has allowed high-fidelity models in hover optimizations, since the cost of the gradient evaluation becomes practically independent of the number of design parameters [4][5]. The optimization problem is more complex in forward flight. The adjoint formulation for unsteady flows requires either considering the problem as periodic to apply a steady adjoint formulation [6] or solving the unsteady adjoint equation backwards in time [7] [8].

The second popular approach for optimization is the application of Genetic Algorithms (GA). The main advantage of GAs is that they can deal with discontinuities and multimodal functions since they do not use derivative information. Furthermore, the optimum point is searched from a large population of points and not only from a single point. The risk of obtaining a local optimum of the objective function is greatly reduced. However, GAs require a significant number of evaluations of the objective function. It is therefore recommended to employ them coupled with a low-fidelity code, which does not consume excessive CPU time. Results from aerodynamic 
optimizations based on the coupling between a comprehensive code and an evolutionary optimizer actually show the effectiveness of this type of optimization procedure to optimize the twist law of a helicopter rotor blade [9].

For two decades, surrogate models, such as the Kriging model, have been developed (initially in the field of spatial statistics and geostatistics) and introduced in optimization procedures for helicopter blade design. Glaz et al. [10] studied the effectiveness of various surrogate models and their accuracy when used in optimization procedures to minimize helicopter vibrations. Among the methods considered (Polynomial Regression, Kriging and Radial Basis Function), the Kriging model appears to be the most effective method to approximate the vibratory loads over the design space, and to reach the optimum design location.

It is now well known that surrogate models are suitable for reducing the computational cost incurred by performing a great number of high-fidelity evaluations, and for enabling the use of high-fidelity simulations in the optimization loop. For instance, CSD/CFD simulations are necessary to take into account fluid-structure interactions [11][12]. Such high-fidelity simulations are especially recommended for complex geometry blades and/or complex flight configurations (moderate to high values of the advancing velocity or rotor thrust). The first step is to consider a single-level fidelity tool to generate a response surface between the objective function and the design variables. Jeong et al. [13] successfully applied a Kriging model to optimize the lift-to-drag ratio of a two-dimensional airfoil. It is shown that replacing a CFD solver by a Kriging model to estimate the objective function in an optimization procedure using GA is very efficient in terms of computational time reduction. Adding the search of the maximum Expected Improvement point is necessary to improve the accuracy of the response surface, as well as to explore the global optimum efficiently. Vu et al [14] proposed a process to obtain an optimal helicopter blade shape for aerodynamic performance in hover flight. A Kriging model was integrated into a computational Model Center tool, in order to quickly predict the optimum design, in conjunction with a gradient-based optimization algorithm. Siguira et al [15][16], within the framework of a cooperation between JAXA and ONERA, proposed an optimization procedure based on the Kriging model, obtained from GA optimization. The Expected Improvement point of the objective function is directly used as a fitness value in the optimization process. GA maximizes the EIs of the objective function, in order to find the non-dominated solutions about the EIs, and several points were selected from the non-dominated solutions to update the Kriging model. This procedure has been used to optimize the twist distribution of a model rotor blade, both in hover and forward flight. 
The second step considers different levels of fidelity to determine the objective function. Collins [17] proposed a multi-fidelity framework combining both low- and high-fidelity tools. The connection between the two models was the application of a scaling operator that multiplies the value obtained by the low-fidelity model. Recent studies performed by Wilke are based on a variable level surrogate model using Hierarchical Kriging [18]. The low- and high-fidelity models were built using dynamic inflow models and Euler equations, respectively. The search of the minimum required power led to optimized blade planforms in hover and in forward flight and resulted in a significant computational cost reduction. This single-objective optimization procedure has been extended to a threelevel fidelity model [19] and to a multi-objective optimization in hover and forward flight [20]. It was shown that this technique allows closer approximation to a reference Pareto front than single-fidelity optimization procedure.

Previously at ONERA, the optimization methods for advancing flight configurations were based on the coupling between a comprehensive analysis code HOST [21] and an optimizer. The performance of the optimized blades was checked a posteriori with CFD calculations, more accurate than two-dimensional simulations to predict the threedimensional and unsteady effects of the flowfield around a rotor blade with a complex geometry. The optimization procedures presented here rely on Kriging and Co-Kriging-based optimization of rotor blades using multi-fidelity methods available at ONERA. These methodologies are described in the first part of the paper. These optimization procedures are applied to define the sweep law of a helicopter rotor blade to improve its performance in forward flight. Aerodynamic analysis of the numerical results obtained for each optimized blade is performed showing benefit of taking into account three-dimensional unsteady effects of the flowfield inside the optimization loop, through Multi-Fidelity surrogate models. More precisely, the origin of the loss or benefit in power will be studied, as well as the aero-elastic behavior of the optimized blades.

\section{Surrogate Model Methodologies}

Surrogate modeling plays an important role in many areas of aerospace engineering, including aerodynamic design optimization, structural design and multidisciplinary optimization. Many methods have been studied, such as polynomial models (RSM), moving least-squares (MLS), Radial Basis Function (RBF), Support Vector Machine (SVM), Kriging and multi-fidelity methods.

Surrogate-model-based optimization is a numerical optimization approach that applies surrogate models to guide the search for the real model optimum, but at a reasonable computational cost. 
ONERA has developed a code, Korrigan, which can build Kriging, gradient- enhanced Kriging and Hessianenhanced Kriging models, as well as Co-Kriging and gradient- enhanced Co-Kriging surrogate models. For all of these approaches, the correlation function can be chosen from the Gaussian or compact polynomial kernels. The correlation function parameters are determined by using a Genetic Algorithm (GA) developed inside Korrigan. This GA also searches for the minimum of the model and maximizes the Expected Improvement (EGO) to update its database.

A model is developed that can approximate the objective function data throughout the parameter space. Sample points are generated using a Design-of-Experiment technique with Latin Hypercube Sampling (LHS) [22]. Improved space-filling properties of the LHS are achieved by minimizing a distance function $\Phi_{p}$ [23]. If the E uclidian distance between any two points is $d_{i j}=\left\|X_{i}-X_{j}\right\|_{2}$, then the criterion is defined by: $\Phi_{p}=\left[\sum_{i=1}^{n-1} \sum_{j=i+1}^{n} d_{i j}^{-p}\right]^{1 / p}$.

In the case of a multi-fidelity model, a sample point set is defined for each fidelity level. The aerodynamic data at sample points are evaluated by the use of respective fidelity level methods. The high-fidelity model is constructed with either the Kriging or Co-Kriging approach. If the termination criterion is not fulfilled, iterative refinement is performed by adding new sample points. These new data are expected to improve the model accuracy and accelerate the search for the optimum.

\section{A. Kriging and EGO Methodology}

Kriging is a statistical interpolation method suggested by Krige [24] and mathematically studied by Matheron [25]. Its estimation depends on spatial correlation between n sample points, $\boldsymbol{X}_{1}, \ldots, \boldsymbol{X}_{n}$, for which the function values have been computed, $\boldsymbol{Y}_{1}, \ldots, \boldsymbol{Y}_{n}$. The Kriging model is a Gaussian process, Z, with covariance function $\operatorname{cov}(.,$. modeled as:

$$
\operatorname{Cov}\left(\boldsymbol{Z}_{i}, \boldsymbol{Z}_{j}\right)=\sigma^{2} \boldsymbol{R}\left(\boldsymbol{Z}_{\mathrm{i}}, \boldsymbol{Z}_{\mathrm{j}} ; \boldsymbol{\theta}\right)
$$

where $\sigma^{2}$ is the variance of the process and $\mathrm{R}$ is a correlation function that depends of internal parameters, $\theta$, which can be determined by optimizing the likelihood.

The spatial correlation function measures the proximity between two points. Many correlation functions can be applied; within the framework of this study, the Gaussian kernel is chosen:

$$
\boldsymbol{R}\left(\boldsymbol{Z}_{\mathrm{i}}, \boldsymbol{Z}_{\mathrm{j}} ; \boldsymbol{\theta}\right)=\exp \left[-\sum_{k=1}^{N} \theta_{k}\left|z_{i}^{k}-z_{j}^{k}\right|^{2}\right]
$$


where $\theta_{k}$ are the correlation internal parameters that control the rate of correlation in the $k^{\text {th }}$ dimension. The $\theta_{k}$ parameters are determined by maximizing the likelihood function:

$$
L=-\frac{N}{2} \ln \left(\sigma^{2}\right)-\frac{1}{2} \ln (|\operatorname{det}(\boldsymbol{R})|)
$$

$L$ is a function of the internal parameters and measures their plausibility given by the observed data.

The optimal unbiased linear predictor provided by the Kriging theory is expressed as:

$$
\hat{Y}(\boldsymbol{X})=m_{Y}+\boldsymbol{r}^{T}(\boldsymbol{X}) \boldsymbol{R}^{-1}\left(\boldsymbol{Y}-\mathbf{1} . m_{Y}\right)
$$

where

$$
\left\{\begin{array}{c}
R_{i j}=\operatorname{corr}\left(\boldsymbol{Z}_{i}, \boldsymbol{Z}_{j}\right) \\
\boldsymbol{r}(\boldsymbol{X})=\left[\operatorname{corr}\left(\boldsymbol{X}, \boldsymbol{Z}_{1}\right), \ldots, \operatorname{corr}\left(\boldsymbol{X}, \boldsymbol{Z}_{n}\right)\right]^{T} \\
\boldsymbol{Y}=\left[Y_{\mathbf{1}}, \ldots, Y_{\boldsymbol{n}}\right]^{T} \\
m_{Y}=\frac{\mathbf{1}^{T} \boldsymbol{R}^{-1} \boldsymbol{Y}}{\mathbf{1}^{\boldsymbol{R}^{-1} \mathbf{1}}}
\end{array}\right.
$$

Kriging provides also an uncertainty estimator (variance) as:

$$
\hat{\sigma}^{2}=\left(\boldsymbol{Y}-\mathbf{1} \cdot m_{Y}\right)^{T} \boldsymbol{R}^{-\mathbf{1}}\left(\boldsymbol{Y}-\mathbf{1} \cdot m_{Y}\right)
$$

The internal parameters $\theta_{k}$ are determined by the use of a genetic algorithm to maximize $L$.

To improve the search for the optimum, it may be necessary to enrich the sampling by adding new points to improve the accuracy of the Kriging model. The selection of these points can be performed by different means: the model minimum point, and the maximum uncertainty point determined by EGO (Efficient Global Optimization) [26]. To improve the model accuracy, the new points must be selected by balanced exploitation and exploration. Thus, EGO uses both the predictor and the variance of the model to estimate the expected improvement (EI) defined by:

$$
E[I(\boldsymbol{X})]=\left(Y_{\min }-\hat{Y}(\boldsymbol{X})\right) \Phi\left(\frac{Y_{\min }-\widehat{Y}(\boldsymbol{X})}{\widehat{\sigma}}\right)+\phi\left(\frac{Y_{\min }-\hat{Y}(\boldsymbol{X})}{\widehat{\sigma}}\right)
$$

where $\Phi($.$) and \phi($.$) are the cumulative distribution function and probability density function, respectively. A new$ selected point corresponds to the maximum of the EI.

\section{B. Co-Kriging Methodology}

The idea of Co-Kriging is to use all available information to estimate unknown high-fidelity information. The basic Kriging formulation has been extended by many authors [27]-[30] to combine multiple levels of simulation to create a more accurate or less expensive high-fidelity model. The Kennedy and O'Hagan approach [27] is based on an autoregressive model and consists in approximating the high-fidelity model by multiplying the low-fidelity 
model, $\boldsymbol{Z}_{\boldsymbol{c}}$, by a scaling factor $\rho$ and by adding a Gaussian process $\boldsymbol{Z}_{\boldsymbol{d}}$ representing the difference between the low and high-fidelity data,

$$
Z_{e}(X)=\rho Z_{c}(X)+Z_{d}(X)
$$

where $\boldsymbol{X}^{T}=\left[\boldsymbol{X}_{c}^{T}, \boldsymbol{X}_{e}^{T}\right], \boldsymbol{X}_{c}$ and $\boldsymbol{X}_{e}$ represent the low and high-fidelity sampling locations. The covariance matrix $C$ is defined by:

$$
\boldsymbol{C}=\left(\begin{array}{cc}
\sigma_{c}^{2} \boldsymbol{R}_{c}\left(\boldsymbol{X}_{c}, \boldsymbol{X}_{c}\right) & \rho \sigma_{c}^{2} \boldsymbol{R}_{c}\left(\boldsymbol{X}_{c}, \boldsymbol{X}_{e}\right) \\
\rho \sigma_{c}^{2} \boldsymbol{R}_{c}\left(\boldsymbol{X}_{e}, \boldsymbol{X}_{c}\right) & \rho^{2} \sigma_{c}^{2} \boldsymbol{R}_{c}\left(\boldsymbol{X}_{e}, \boldsymbol{X}_{\boldsymbol{e}}\right)+\sigma_{d}^{2} \boldsymbol{R}_{d}\left(\boldsymbol{X}_{e}, \boldsymbol{X}_{e}\right)
\end{array}\right)
$$

The correlation functions are written in a similar way to those for the Kriging methodology, and they require that twice the number of the internal parameters to be determined. Given that $\boldsymbol{Z}_{\boldsymbol{c}}$ and $\boldsymbol{Z}_{\boldsymbol{d}}$ are considered to be independent, the internal parameters of the low-fidelity model can be determined in a similar manner to those of the Kriging model. Thus $\rho$ and the internal parameters of the difference process can be determined by optimizing the likelihood, but using the difference data:

$$
\boldsymbol{d}=\boldsymbol{y}_{e}-\rho \boldsymbol{y}_{c}\left(\boldsymbol{X}_{e}\right)
$$

so that the predictor provided by Co-Kriging is now expressed as:

$$
\hat{Y}_{e}(\boldsymbol{X})=m_{Y}+\boldsymbol{c}^{T}(\boldsymbol{X}) \boldsymbol{C}^{-1}\left(\boldsymbol{Y}-\mathbf{1} \cdot m_{Y}\right)
$$

where

$$
\left\{\begin{array}{c}
Y^{T}=\left[Y_{c}^{T}, Y_{e}^{T}\right] \\
m_{Y}=\frac{\mathbf{1}^{T} C^{-1} Y}{\mathbf{1}^{T} C^{-1} \mathbf{1}}
\end{array}\right.
$$

Kriging also provides an uncertainty estimator (variance) and an estimated mean squared error defined as:

$$
\left\{\begin{array}{c}
{\widehat{\sigma_{d}}}^{2}=\left(\boldsymbol{Y}-\mathbf{1} \cdot m_{Y}\right)^{T} \boldsymbol{C}^{-1}\left(\boldsymbol{Y}-\mathbf{1} \cdot m_{Y}\right) \\
\widehat{S}^{2} \approx \rho^{2}{\widehat{\sigma_{c}}}^{2}+{\widehat{\sigma_{d}}}^{2}-\boldsymbol{c}^{T} \boldsymbol{C}^{-1} \boldsymbol{c}+\frac{1-\mathbf{1}^{T} \boldsymbol{C}^{-1} \boldsymbol{c}}{\mathbf{1}^{T} \boldsymbol{C}^{-1} \mathbf{1}}
\end{array}\right.
$$

Normalized Root Mean Square Error (NRMSE) on a validation data set of $n_{p}$ uniformly distributed points is used as error metric to asses the surrogate model accuracy. The error metric is expressed as:

$$
N R M S E=\frac{\sqrt{\sum_{i=1}^{n_{p}} \frac{\left(y_{t}^{i}-\widehat{y^{\imath}}\right)}{n_{p}}}}{\max \left(y_{t}\right)-\min \left(y_{t}\right)}
$$

where $y_{t}$ is the vector of true response values. 


\section{Validation on an analytical function}

The advantages of the Co-Kriging method are illustrated by the one-dimensional "benchmark" problem given in Forester et al.[28]. The data are defined by the analytic functions:

$$
\begin{gathered}
Y_{l f}=\frac{1}{2} Y_{h f}+10(x-1) \\
Y_{h f}=(6 x-2)^{2} \sin (12 x-4)
\end{gathered}
$$

The four high-fidelity sample data, $X_{h f}=\{0,0.33,0.66,1.0\}$ usually chosen for this test case are not sufficient to provide an accurate Kriging model. Fig. 1 illustrates the true function and the Kriging model built from the $X_{h f}$ data. Low-fidelity sample data is used $X_{l f}=\{0,0.1,0.2,0.3,0.4,0.5,0.6,0.7,0.8,0.9,1.0\}$. The Kriging model built from the $X_{l f}$ data and the Co-Kriging model built with the two sample data are also depicted in Fig. 1. When the highfidelity data are added to the low-fidelity data, the data are now sufficient for the Co-Kriging theory to improve the model. The true function and the Co-Kriging model match very well. It must be noted that, in this case, the very good accuracy obtained is due to the fact that the two functions correspond to the autoregressive theoretical model.

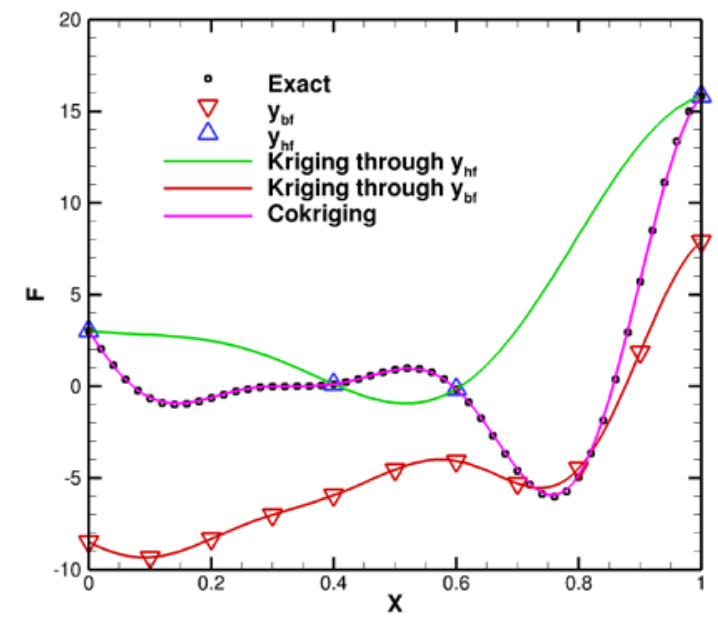

Fig. 1: One-variable example of Kriging and Co-Kriging.

The error metric evaluated with $n_{p}=1001$ is $N R M S E=0.0705$, which is a very satisfactory value.

\section{Design of Experiments}

As previously discussed, the Design of Experiment is built with an improved LHS method. A two-dimensional test case is defined from the McCormick function [31]:

$$
\begin{gathered}
Y_{l f}=\sin \left(x_{1}+x_{2}\right)+\left(x_{2}-x_{1}\right)^{2} \quad x_{1} \in[-1.5,4] \\
Y_{h f}=Y_{b f}-1.5 x_{1}+2.5 x_{2}+1 .
\end{gathered}
$$


where $\mathrm{Y}_{\mathrm{lf}}$ is the analytical function representing the low-fidelity level, $\mathrm{Y}_{\mathrm{hf}}$ the high-fidelity level. The minimum value of $Y_{\text {hf }}(-1.9133)$ is located at the point $(-0.54719,-1.54719)$, but the minimum value of $Y_{b f}(-1)$ is located at $(\pi / 4,-\pi / 4)$. A comparison between the improved LHS and Hammersley sampling methods for 16 samples (Fig. 2) yields equivalent in-fill sampling properties and similar low-fidelity Response Surface Models.
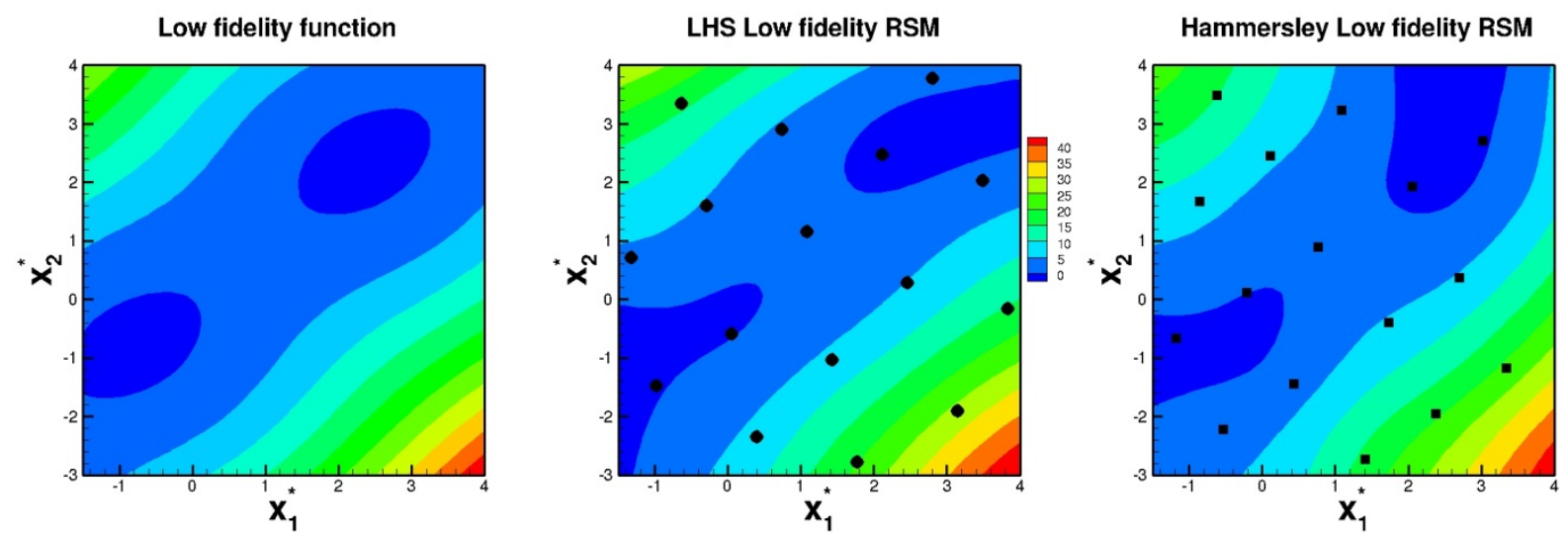

Fig. 2: Comparison between LHS and Hammersley Low-Fidelity RSM (16 samples)

The sensitivity to the HF and LF samplings has been studied. To start the optimization, the Co-Kriging RSM is built with $N_{h f}$ and $N_{l f}$ high and l ow-f idelity point s. Durin g the o ptim ization proce ss, at ea ch itera tion the model minimum point and the maximum EI point are determined. Several sampling number associations have been evaluated: $N_{h f} \in\{4,5\}$ and $N_{l f} \in\{8,10,12,14,16\}$. For each association, the total process is repeated 30 times, with a different Design of Experiment sampling generated each time. The mean curves (Fig. 3) have the same global properties confirming repeatability. After 15 and $20 \mathrm{HF}$ evaluations the error is, respectively, of order $10^{-3}$ and $10^{-4}$. The convergence rate is greatly decreased with additional HF sampling. A slight improvement is observed when 5 HF samples are used rather than 4, but this trend is not significant. The insensitivity to the LF sampling is due to the fact that in optimization, a global accuracy of the LF model is not necessary. A comparison with a mean convergence curve obtained with a Kriging optimization starting with 10 LHS sampling demonstrates the accuracy benefit obtained for the same number of HF evaluations. These conclusions and orders of magnitude are valid for cases where the number of parameters is very small. 


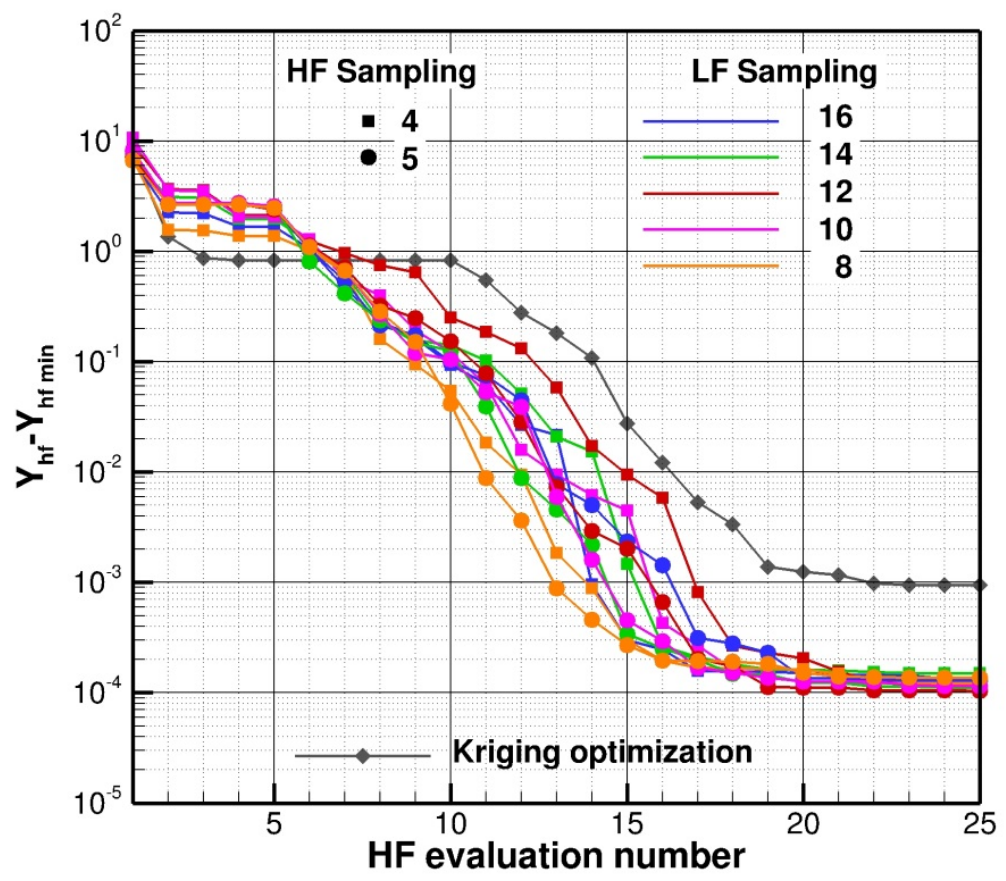

Fig. 3: Convergence curves with respect to $\mathrm{HF}$ evaluation number for different $\mathrm{N}_{\mathrm{hf}}$ and $\mathrm{N}_{\text {If }}$ associations

\section{Application to Rotor Blade Optimization}

The objective of this study is to apply the previously described Kriging and Co-Kriging optimization procedures to optimize the sweep of a reference rotor defined by Airbus Helicopters Deutschland to improve its performance in forward flight. This rotor is equipped with five blades with an aspect ratio of 18.6. Each blade is rectangular with a parabolic blade tip. The blade is formed from the two airfoils OA312 and OA309, where linear interpolation defines the transition between these airfoils. A linear geometric twist is defined, and no anhedral is applied at the blade tip. The planform of the reference blade is illustrated in Fig. 4.

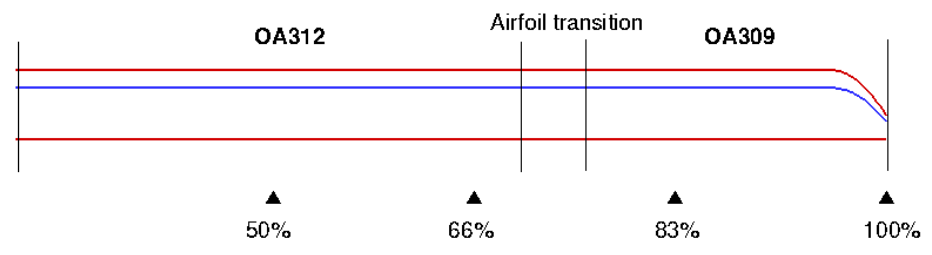

Fig. 4: Reference blade planform and location of control points 
The objective function is the minimization of the total power consumed by the main rotor for a selected forward-flight configuration. No constraints are considered. Three active decision variables on the sweep are chosen, located at $66 \%, 83 \%$ and $100 \%$ of the rotor radius $\mathrm{R}$. These variables are parameterized by cubic splines, whose control point locations are identified in Fig. 4. The first control point defining the cubic spline is located at 50\% of the rotor radius, and is imposed at zero, in order to ensure a smooth transition between the initial area (up to 50\%) and the optimized area (from 50\%). The lower and upper bound values are respectively set at $0.20 \mathrm{~m}$ and $0.50 \mathrm{~m}$.

The selected forward-flight speed $V_{h}$ is equal to $140 \mathrm{kts}$, and the rotational velocity $\Omega$ is $36.34 \mathrm{rad} / \mathrm{s}$ (347 rpm), which corresponds to an advance ratio $\mu$ of 0.36 . To correctly perform performance assessment, it is necessary to trim the rotor at a prescribed propulsive force. A three-variable objective trims the rotor at prescribed values for lift $\left(\mathrm{C}_{\mathrm{T}} / \sigma=0.075\right)$, propulsive force $\left(\left(\mathrm{C}_{\mathrm{D}} \mathrm{S}\right)_{\mathrm{f}} / \mathrm{S} \sigma=0.15\right)$, and rolling moment $\left(\mathrm{M}_{\mathrm{x}}=0 \mathrm{Nm}\right)$. The shaft angle is then prescribed as equal to the arctangent of the ratio between the drag and the thrust coefficients. With this trim approach, the hub pitching moment reaches reasonable negative values given the prescribed setting of the shaft angle.

\section{E. Optimization chains}

The search for the optimized solution using a single-fidelity model based on Kriging methodology is performed following the optimization procedure represented in Fig. 5. 


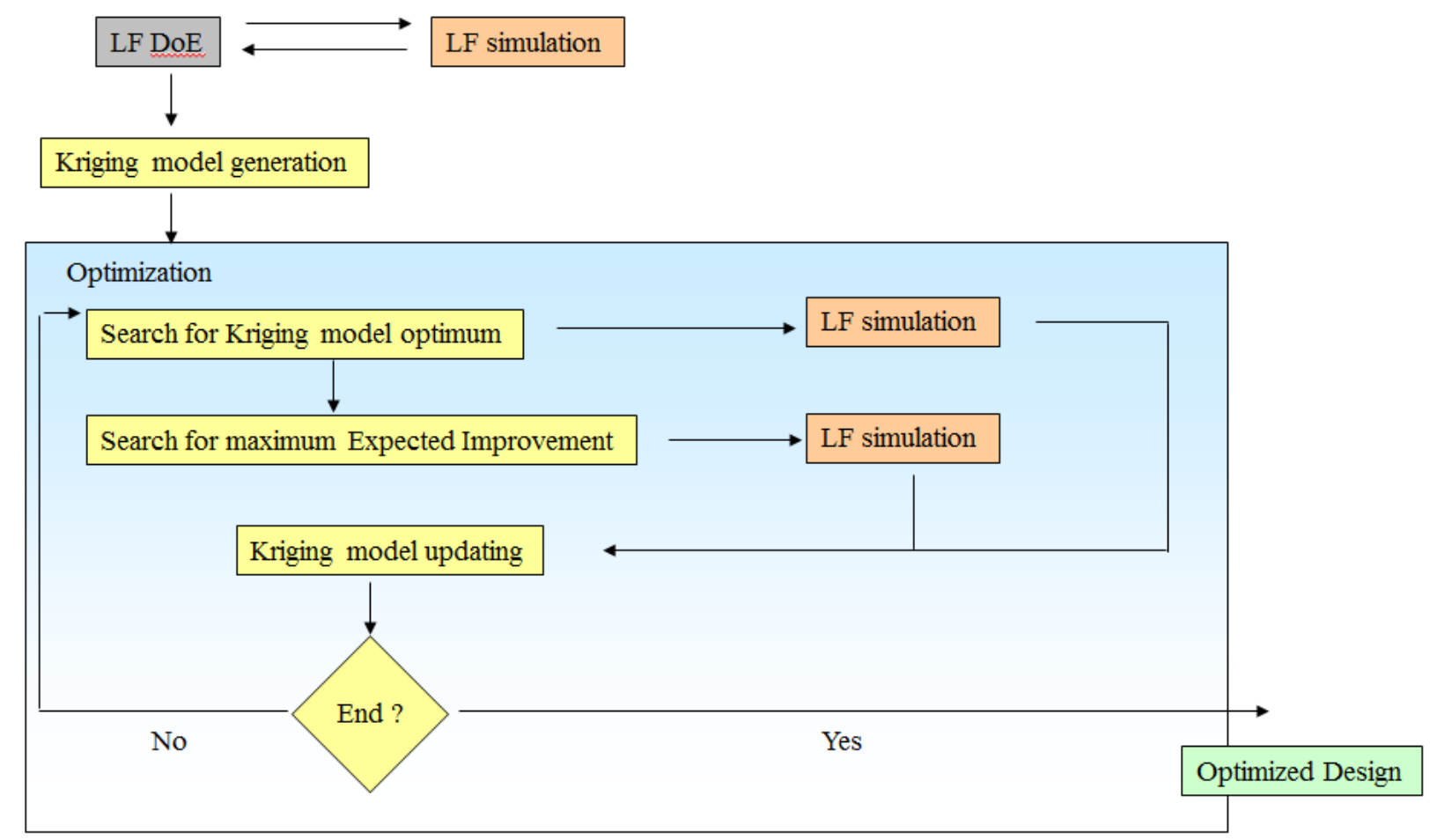

Fig. 5: Chart of the optimization procedure by Kriging.

The first step consists in building the Design of Experiment (DoE), based on Latin Hypercube Sampling spatial discretization of the design space. Then, the generation of the Kriging model is divided into two steps: the search for the minimum point of the model obtained using a classical genetic algorithm optimizer, followed by the search for the maximum Expected Improvement. The Design of Experiment is enriched at each step by Low-Fidelity (LF) simulations of these points. This procedure is repeated until a prescribed number of evaluations is completed, and a check is carried out to verify that the power benefit cannot be improved.

The optimization procedure based on the multi-level Co-Kriging model is shown in Fig. 6. 


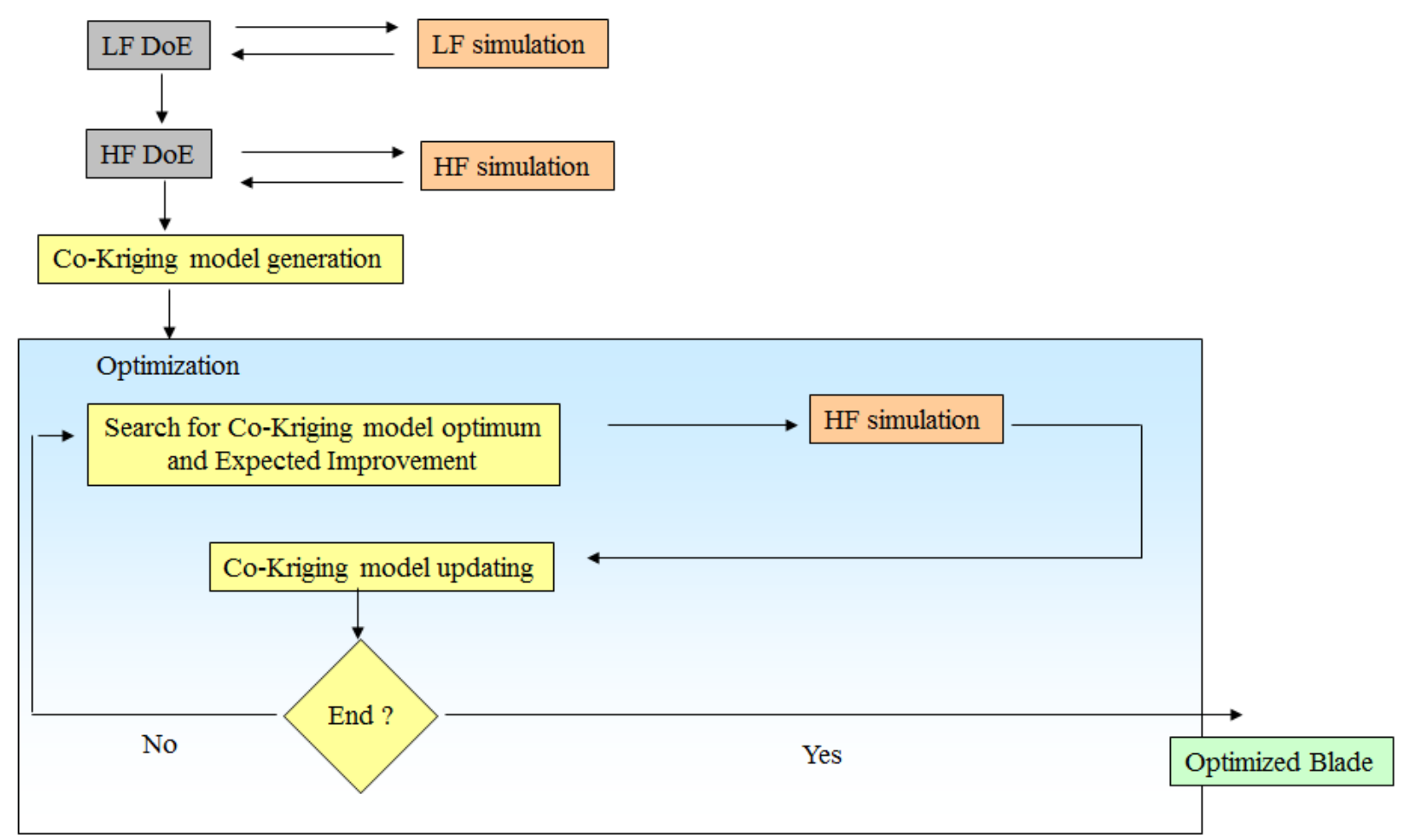

Fig. 6: Chart of the optimization procedure by Co-Kriging.

The first step consists in the generation of the two Design of Experiment databases: the first one defining the same LF model built for the Kriging approach now using a moderate number of points; the second one defining the High-Fidelity (HF) model built from a restricted number of points. Then the generation of the Co-Kriging model begins. The model Minimum point and the model maximum Expected Improvement point are sought by using the genetic algorithm. To limit the number of very expansive High-Fidelity evaluations, only the Minimum point is evaluated by HF simulations when this point and the Expected Improvement point are considered to be close to each other. Otherwise both Minimum point and Expected Improvement point are evaluated by HF simulations. The new point(s) is (are) then added to enrich the data base. Another optimization loop can begin until the optimized solution converges. Convergence is determined by the user.

\section{F. Numerical Tools for Low-Fidelity Simulations}

The Low-Fidelity simulations are performed with the HOST (Helicopter Overall Simulation Tool) comprehensive rotor code, developed by Airbus Helicopters [21]. 
The structural model is based on a one-dimensional Euler-Bernoulli beam model. The beam is discretized along the pitch axis as an assembly of rigid segments with the elastic properties contained in the joints connecting them. The structural properties of the blade (mass, inertia, stiffness per unit length, etc) are given as an input data file for HOST. During the aerodynamic optimization, the blade planform is modified, leading to a change of these structural data. In 2011, ONERA defined an updating procedure for the structural data used in the HOST code that can be integrated into an optimization loop [9]. This procedure is based on the definition of analytical polynomial laws that describe the evolutions of the stiffnesses, the distribution of the mass, and the inertia per unit length with respect to the chord and the thickness distributions of the profiles of the blade. Some analytical corrections are also performed to adjust the elastic axis and the gravity center axis with respect to the pitch axis. This procedure allows realistic blade planforms to be obtained with internal structural properties suitable for the new blade design. This procedure has been employed within the framework of this study for the optimization of the sweep law of the selected reference rotor.

The HOST comprehensive code is based on a lifting line approach to compute the blade aerodynamic loads. The blade is considered as a succession of two-dimensional blade elements, each one shedding a vortex of bound circulation generated at its aerodynamic center (quarter-chord). At a given Mach number and for an equivalent angle of attack, the lift, drag and pitching moment coefficients can be obtained via two-dimensional semiempirical airfoil lookup tables. The compressibility effects and the viscosity are partially assessed in these tables. Furthermore, numerical corrections can be applied to include the effects of the blade geometry such as sweep and curvature, the effects of rotation, and the effects of dynamic stall and unsteadiness. Sweep corrections modify the incidence and the Mach number with respect to the oblique attack of the wind. Curvature effects allow operating conditions of the airfoils in the plane perpendicular to the quarter-chord line of the profiles. These geometrical corrections increase the accuracy of the lifting line theory and are suitable for moderate evolutive blade planforms.

The wake influences the rotor performance via the induced velocities that it generates at the rotor disk. Within the framework of this study, the Airbus Helicopters METAR [32] prescribed-wake model is used. The wake geometry is prescribed and considered helical. The induced velocities generated by a vortex segment of the wake are computed using the Biot-Savart law. This system is solved iteratively in the trim loop until convergence is obtained, when the circulation of the wake is in accordance with the sectional blade lift forces, and when the mean induced velocity reaches a threshold value. 
HOST computations provide the rotor trim characteristics such as the control commands (pitch, flap and lag angles), the local aerodynamic loads, the blade elastic deformations, and the shaft power (which is the objective function of this study). The CPU cost of one evaluation is between 2 and 5 minutes on a single processor personal computer.

\section{G. Numerical Tools for High-Fidelity Simulations}

The HF simulations are performed using a loose coupling procedure [33] between the CSD code, HOST, and the CFD code developed at ONERA, elsA [34]. The three-dimensional unsteady Navier-Stokes equations are solved by the cell-centered second-order Jameson scheme. The time integration is performed by an implicit Euler scheme with Gear sub-iterations. The time step is equivalent to $1.2^{\circ}$ of blade rotation. The turbulence model is Kok k- $\omega$ [35] with Menter Shear-Stress Transport (SST) corrections [36], as the flow is assumed to be fully turbulent. The grids are generated using the Chimera technique. A multi-block, deformable mesh of $\mathrm{O}-\mathrm{H}$ type is generated around each blade containing 1.7 million points. These blade grids are immersed in a Cartesian background grid containing 13 million points. Based on prior experience, total mesh of 21.5 million points can be considered as refined [37].

To obtain a satisfactory level of convergence of the coupling procedure between the CSD and CFD codes, six iterations are performed for each design point. The CPU cost for a converged coupling procedure is about 90 hours, on 64 processors of the ONERA SGI parallel calculator.

\section{H. Analysis of Optimization Results with Kriging}

The first step of the Kriging optimization procedure is to build the Design of Experiment database. Over the initial 30 points defined by the Latin Hypercube Sampling procedure, 16 points have reached the numerical convergence of the Low-Fidelity simulations performed with the HOST code. Then, the database has been enhanced by the search for the optimum points followed by the search for the Expected Improvement points. After 47 converged HOST evaluations, the Kriging optimization process converged to the global optimum solution (Fig. 7). 


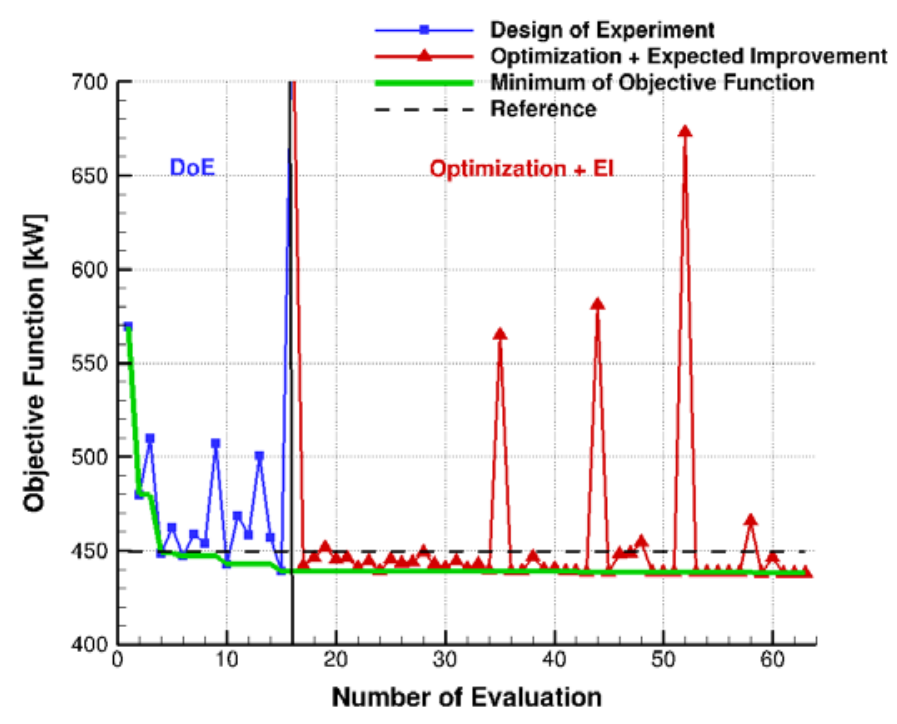

Fig. 7: Convergence of the Kriging optimization procedure.

This solution is similar (Fig. 8) to that obtained with the CMA-ES evolutionary optimizer [38], but with a large reduction of the HOST numerical evaluations. The CPU cost is now reduced by a factor of 5 . The power benefit with respect to the reference blade is equal to $2.5 \%$.

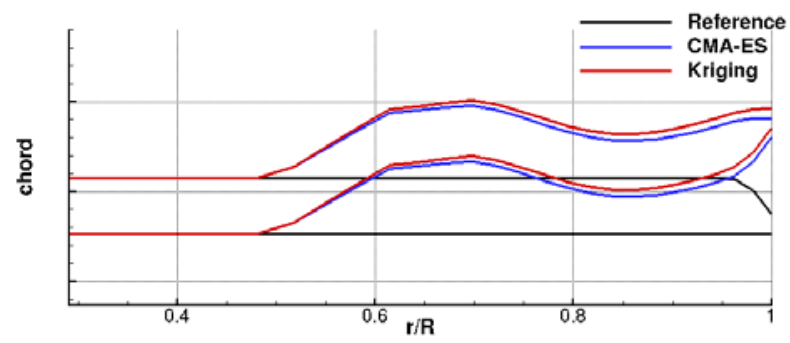

Fig. 8: Optimized blade planform obtained by CMA-ES and Kriging procedures (with updated structural data).

The optimization procedures lead to a modification of the sweep as the optimized blade has a forward sweep of $50 \%$ to $73 \%$ of the span, followed by a backward sweep of $73 \%$ to $90 \%$ of the span, and finally a reduced forward sweep up to the tip. This optimized blade planform is less sinuous, with reduced sweep angles in the backward and the forward directions when compared to a blade optimized without updating structural data during the optimization procedure.

The optimization procedure leads to a benefit of $2.5 \%$ on the total power. The comparison between the reference and optimized Kriging rotors of the control angles and the mean elastic tip twist predicted by HOST calculations is given in Table 1. 


\begin{tabular}{|c|c|c|c|c|}
\hline Rotor & $\theta_{0}\left(^{\circ}\right)$ & $\left.\theta_{\mathrm{el}}{ }^{\circ}\right)$ & $\theta_{1 \mathrm{c}}\left({ }^{\circ}\right)$ & $\theta_{1 \mathrm{~s}}\left({ }^{\circ}\right)$ \\
\hline Reference & 13.83 & -2.98 & 0.53 & -7.97 \\
\hline Optimized with Kriging & 10.89 & 0.68 & 0.11 & -5.64 \\
\hline
\end{tabular}

Table 1: Comparison of control angles and mean elastic tip twist (LF calculations)

The effect of the control angles can be analyzed on the distribution of the local lift coefficient on the rotor disk, for the two rotors, as shown in Fig. 9.
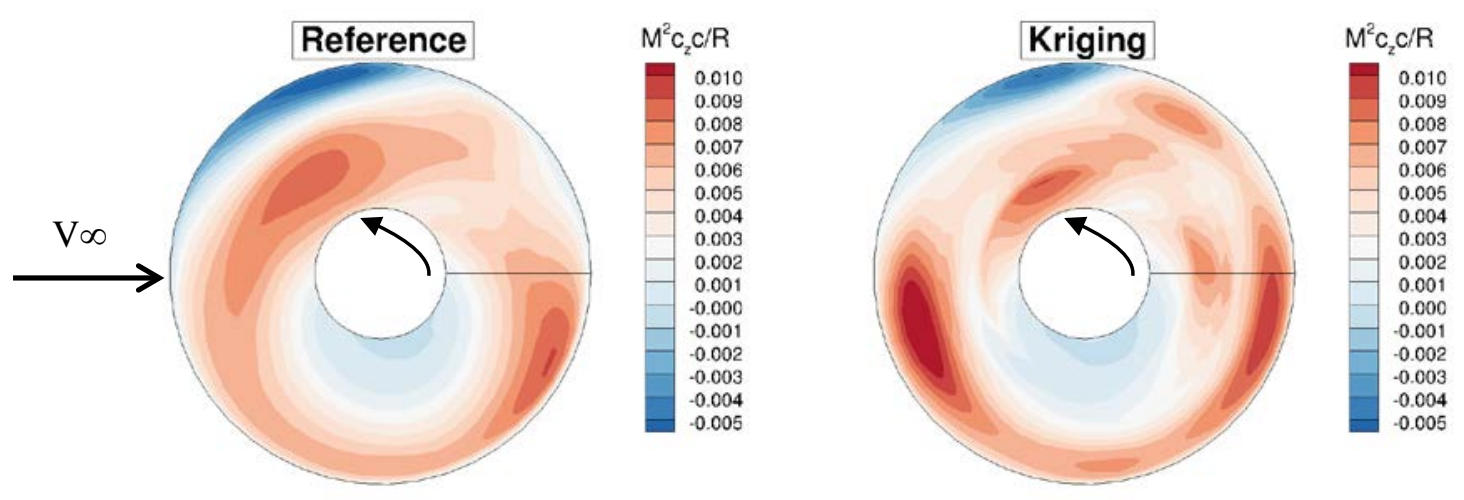

Fig. 9: Distribution of the sectional lift coefficient on the reference and Kriging optimized blades (LF
calculations)

The HOST low-fidelity calculations for the Kriging optimized blade predict a reduction of the lifting loads in the inner part of the rotor disk during the second quarter, between $90^{\circ}$ and $180^{\circ}$ of azimuth, with respect to the reference, balanced with an increase of the lifting loads at the tip of the front blade, between $180^{\circ}$ and $210^{\circ}$ of azimuth. These discrepancies can be explained by the influence of the pronounced backward sweep on the outer part of the blade. The rotation plane is more forwardly inclined by the variation of the $\theta_{1 \mathrm{~s}}$ longitudinal cyclic pitch $\left(+2.3^{\circ}\right)$, and increasing the local loads in the front blade. The different breaks in the sweep distribution of the optimized blade lead to a discontinuous airload distribution on the rotor disk predicted by the HOST code.

The power assessment predicted by HOST reduces required power by an $11 \%$ reduction of the induced power (defined as the scalar product between the aerodynamic force vector and the induced velocity vector). The variations of the sectional lift coefficient and the vertical induced velocity distribution between the optimized Kriging and the reference rotors have a direct effect on the surface distribution of the induced power on the rotor disk, as illustrated in Fig. 10. 

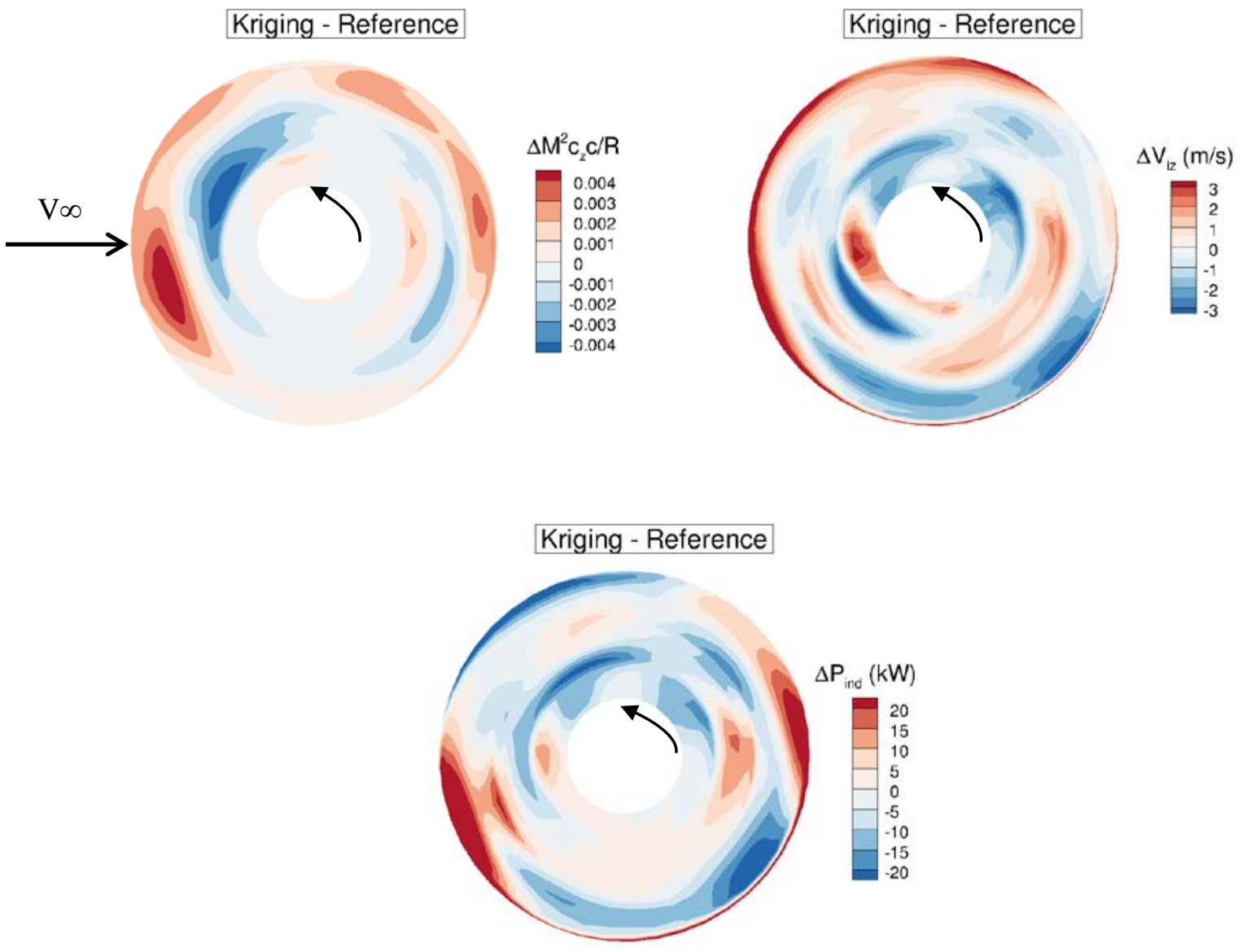

Fig. 10: Distribution on the rotor disk of the difference (Kriging - Reference) of sectional lift coefficient, vertical induced velocity, and surface induced power (LF calculations)

The primary areas of induced power reduction occurs (blue areas) mainly correspond to the locations of reduced values of the vertical induced velocity, located in the inner part of the advancing blade, around the azimuth of $90^{\circ}$, at the blade tip near the azimuth of $300^{\circ}$, and in the fourth quadrant. The areas where a gain in the induced power is predicted for the Kriging optimized blade with respect to the reference are predominantly in regions where an increase in the induced power occurs (red areas), especially at the tip of the front blade, corresponding to the rotor disk location where the lifting loads are largely increasing.

The blade sweep optimization also has an influence on the elastic torsion deformation and on the flap displacement at the blade tip, as observed in Fig. 11. 

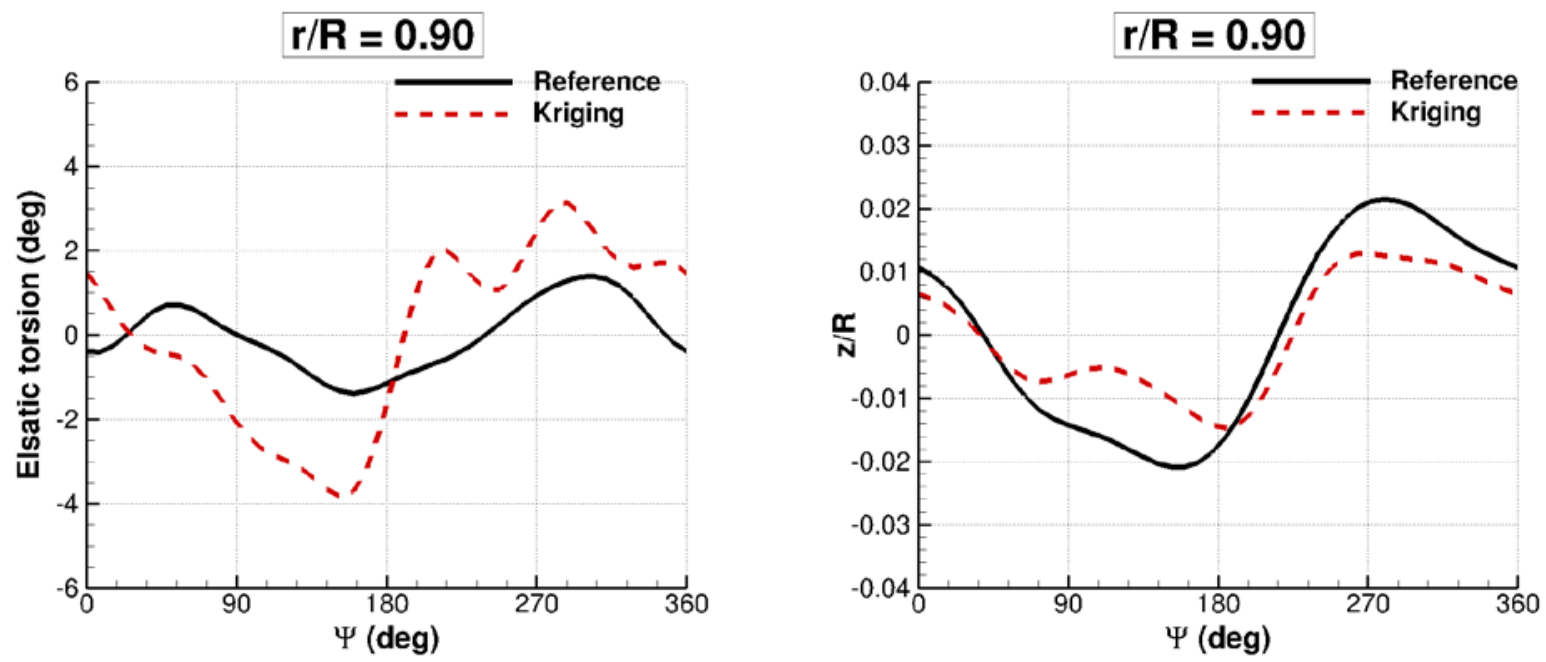

Fig. 11: Elastic torsion and dimensionless flap deformations (mean removed) near the blade tip for the reference and the Kriging optimized blades (LF simulations).

Due to its forward sweep planform, the optimized blade obtained by the Kriging optimization procedure has a higher elastic torsional response than the reference in terms of amplitude (about $4^{\circ}$ instead of $2^{\circ}$ ). The increase of the torsion amplitude can be linked to the reduced value of the collective pitch angle. From Table 1 the decrease of the collective pitch angle compensates for the increase of the mean elastic twist at the tip. A strong steady twist is equivalent to a reduction of the collective pitch angle. The sum of these two values is of the same order of magnitude for both rotors. The flap displacement is less sensitive than the torsion angle in the blade optimization when applying a similar evolution.

\section{Analysis of Optimization Results with Co-Kriging}

In the Kriging optimization process, the first step is to evaluate the objective function (shaft power consumed by the main rotor) with the High-Fidelity numerical tool over a very limited number of points to build the High-Fidelity Design of Experiment. Four points have been chosen among the 30 initial ones resulting from the Latin Hypercube Sampling procedure. 


\begin{tabular}{|c|c|c|}
\hline Blade planform & $\%(\mathrm{LF})$ & $\%(\mathrm{HF})$ \\
\hline LHS2 & +14.2 & +36.7 \\
\hline LHS4 & +1.5 & -0.3 \\
\hline LHS7 & +2.7 & +2.4 \\
\hline LHS9 & +4.1 & +0.4 \\
\hline Optim Kriging & & $+\mathbf{0 . 7}$ \\
\hline
\end{tabular}

Fig. 12: Blade planforms and power benefits estimated by LF and HF simulations for the HF DoE and the optimized rotor by Kriging.

It is very interesting that the hierarchy between the different rotors can differ with respect to the level of fidelity of the numerical tools (Fig. 12). In particular, the HF simulations predict a loss of performance for the optimized blade resulting from the Kriging procedure. The HOST lifting line theory is not accurate enough to correctly predict the performance of blades with a pronounced curvature in geometry, especially with significant sweep at the tip. Three-dimensional unsteady effects can have a major influence on rotor blades, especially those designed with a double sweep. Hence it is important to include these effects in the optimization procedure by using surrogate models, such as Co-Kriging, and supplying CFD computations.

The convergence of the optimization procedure with Co-Kriging is illustrated in Fig. 13. Only the evaluations of the different Minimum points are presented. 


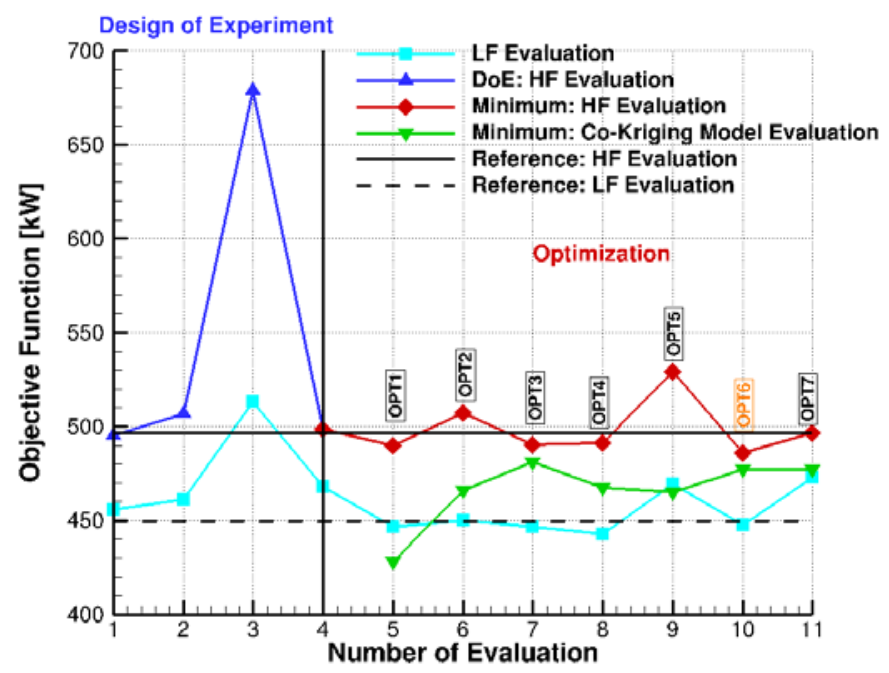

Fig. 13: Convergence of the optimization procedure with Co-Kriging (Minimum points only presented)

$12 \mathrm{HF}$ simulations are required to obtain an optimized solution: four for the generation of the Design of Experiment, 7 for the search for the Minimum Point, and 1 for the search for the Expected Improvement point. This demonstrates the efficiency of the Co-Kriging method in reaching an optimum point that requires at minimum 60 to 70 evaluations with the Kriging method. The gain in CPU time with the Co-Kriging method is approximately a factor of 6 with respect to the Kriging method.

Until the $6^{\text {th }}$ evaluation, the minimum point and the maximum Expected Improvement point of the Co-kriging model are very close. Therefore, only HF evaluations are performed on the Minimum points. This is done to avoid the correlation matrix from being ill-conditioned, highlighted by a sharp increase of the condition number. For the OPT6 point, the difference between the minimum of the model and the estimation of this minimum point by HF calculations is less than $2 \%$, which is considered to be satisfactory for design.

Beyond the OPT6 point, the Expected Improvement EI6 point (for which a significant difference appears with respect to the OPT6 point) and the next OPT7 optimization point have been sought by the procedure, and then evaluated by HF calculations. As shown in Fig. 14, the EI6 design does not provide any power benefit estimation. The estimated power benefit provided by the OPT7 optimized design is lower than that obtained by the OPT6 point, which can be considered the best optimization point of the Co-Kriging procedure. 


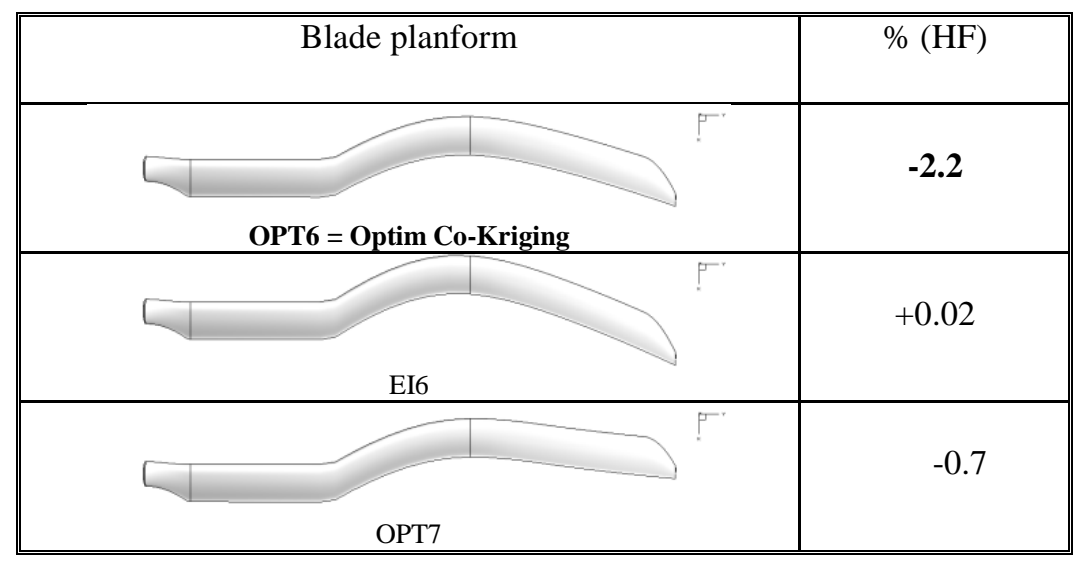

Fig. 14: Blade planforms and power benefits estimated by HF simulations during the Co-Kriging optimization procedure.

The Co-Kriging optimization procedure results in a blade planform presenting a forward and then a backward sweep, evolving in a smoother manner than the blade planform generated by the Kriging optimization. The sweep angles are also reduced with the Co-Kriging optimization procedure. Thus, this blade planform appears more realistic than the optimized Kriging blade. The sweep break for the optimized blade planforms corresponds to the location of the first optimized control point (at mid-span), where the sweep distribution is parameterized by a cubic spline. These designs have been generated within the framework of a theoretical study and should be adapted if necessary.

The shapes of the models obtained by Kriging and Co-Kriging optimization procedures, from which the respective optimum points were obtained, are plotted against the three dimensionless decision variables (defined between 0 and 1) in Fig. 15.
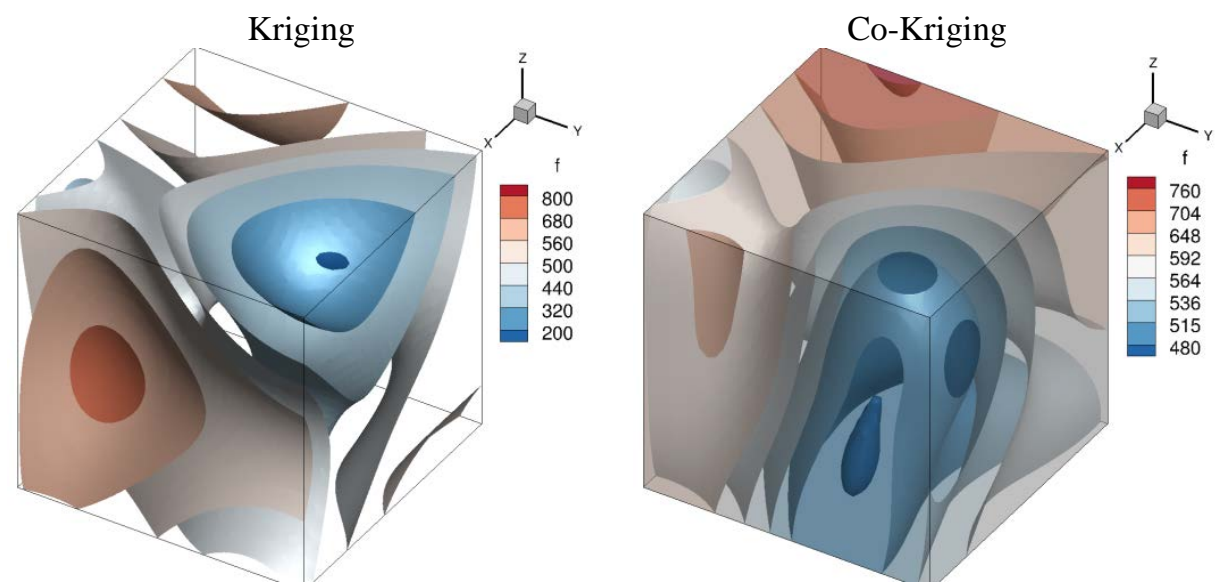

Fig. 15: Shapes of the Kriging and the Co-Kriging models. 
Three zones can be detected on the Kriging and the Co-Kriging models: two with a maximum level and one with a minimum level. However, their shapes are quite different. For the Kriging model, the minimum point is inside an area close to the upper boundary of the search domain. For the Co-Kriging model, the minimum point is detected in a more extended region inside the domain. The Co-Kriging domain results in a large modification of the shape of the surface response and not only an improvement of the accuracy of the Kriging model.

The values of the control angles predicted by HF simulations for the reference and the optimized rotors obtained by Kriging and Co-Kriging models are given in Table 2.

\begin{tabular}{|c|c|c|c|c||}
\hline Rotor & $\theta_{0}\left(^{\circ}\right)$ & $\theta_{\mathrm{el}}\left(^{\circ}\right)$ & $\theta_{1 \mathrm{c}}\left({ }^{\circ}\right)$ & $\theta_{1 \mathrm{~s}}\left({ }^{\circ}\right)$ \\
\hline Reference & 13.98 & -3.09 & 1.17 & -8.07 \\
\hline Optimized with Kriging & 10.98 & 1.09 & 1.01 & -6.26 \\
\hline Optimized with Co-Kriging & 13.58 & -1.76 & 1.91 & -9.55 \\
\hline
\end{tabular}

\section{Table 2: Comparison of control angles and mean elastic tip twist (HF simulations)}

Significant discrepancies are noticeable between the two optimized blades, especially in terms of the longitudinal cyclic pitch angle. Once again, the increase of the mean elastic twist at the blade tip is equivalent to a reduction in the collective pitch control.

The power benefit for the optimized blade obtained with the Co-Kriging model with respect to the reference rotor is estimated at $2.2 \%$ by High-Fidelity computations, which is very encouraging. The different optimized blade planforms lead to modifications of the sectional lift distribution on the rotor disk, estimated by the HF simulations (Fig. 16) for the reference and the optimized rotors resulting from the Kriging and the Co-Kriging procedures, respectively. 


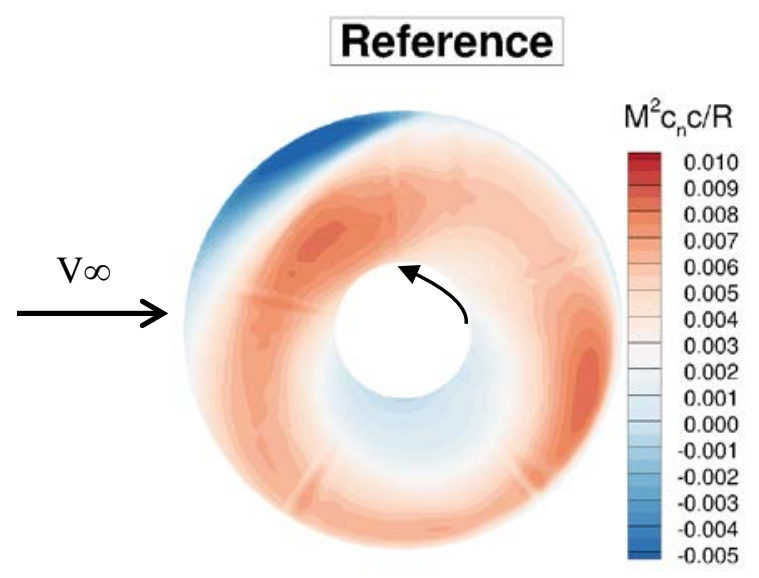

\section{Optim Kriging}

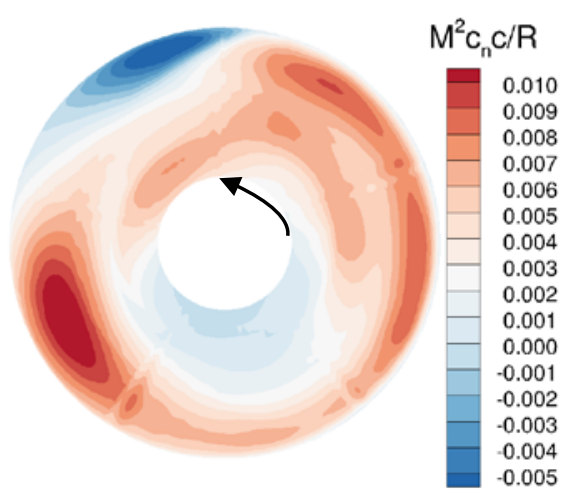

\section{Optim Co-Kriging}

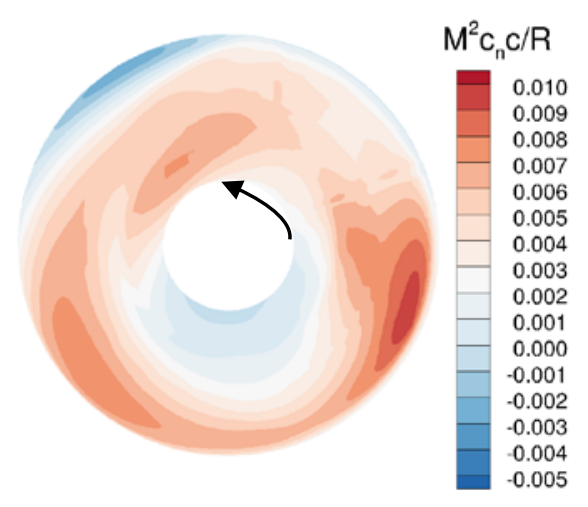

Fig. 16: Distribution on the rotor disk of the sectional lift coefficient for the reference, the Kriging and the Co-Kriging optimized rotors (HF simulations).

The increased negative value of the longitudinal cyclic pitch angle for the Co-Kriging optimized blade with respect to the Kriging blade (-3.3 $)$ (Table 2) can explain the vanishing of the over-loaded area at the blade tip in the front region, as no backward sweep evolution is predicted. The local lift is then increased at the rear part of the CoKriging optimized blade. A reduction of the negative peak of the lifting loads at the blade tip in the second quadrant is also noticeable. The optimization of the sweep by the Co-Kriging procedure, taking into account the threedimensional effects, leads to a smoother distribution of the sectional lift coefficient than for the reference and the Kriging- optimized blades. This distribution of the lifting loads with reduced peak to peak variations can explain the benefit of the total power. 
The influence of the optimized blade planforms on the distribution of the local drag coefficient over the rotor disk, between the reference, the Kriging, and Co-Kriging optimized blades, predicted by HF calculations, can be analyzed in Fig. 17.
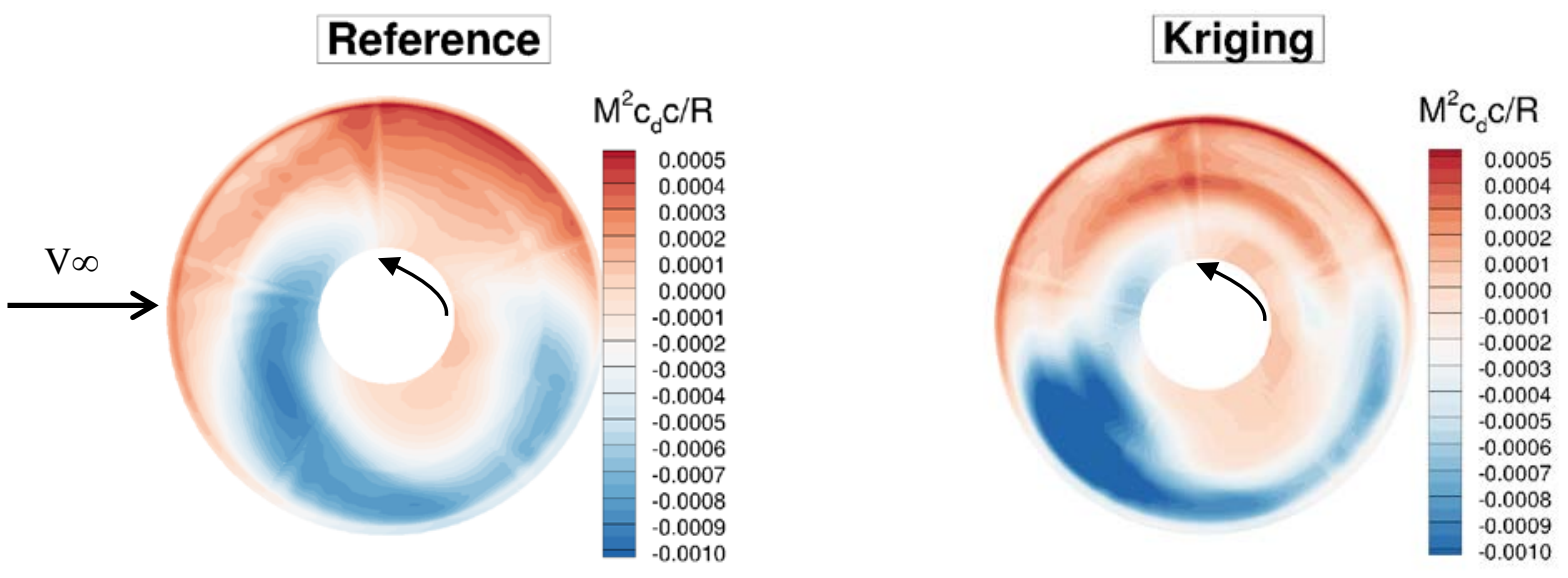

\section{Co-Kriging}

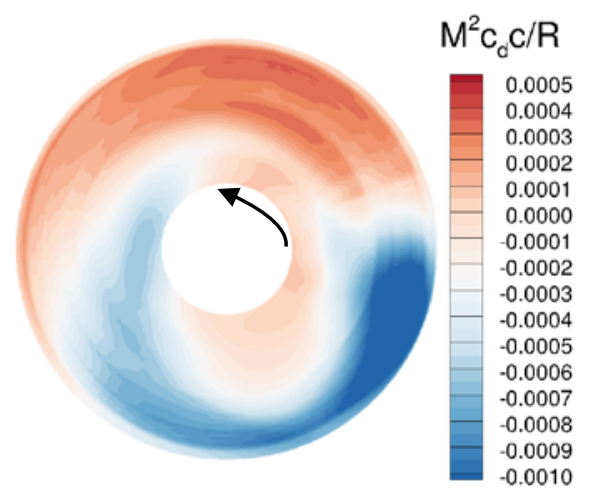

Fig. 17: Distribution on the rotor disk of the sectional drag coefficient for the reference, the Kriging, and the Co-Kriging optimized rotors (HF simulations)

For the Co-Kriging optimized blade, the positive values of the drag coefficient are reduced in the first and second quadrants, and the negative values are less negative in the third quadrant with respect to the reference and the Kriging optimized blades. This distribution of the drag loads can be beneficial to reduce the total power.

The blade tip deformations in torsion and flap obtained by the reference and two optimized rotors are examined in Fig. 18. 

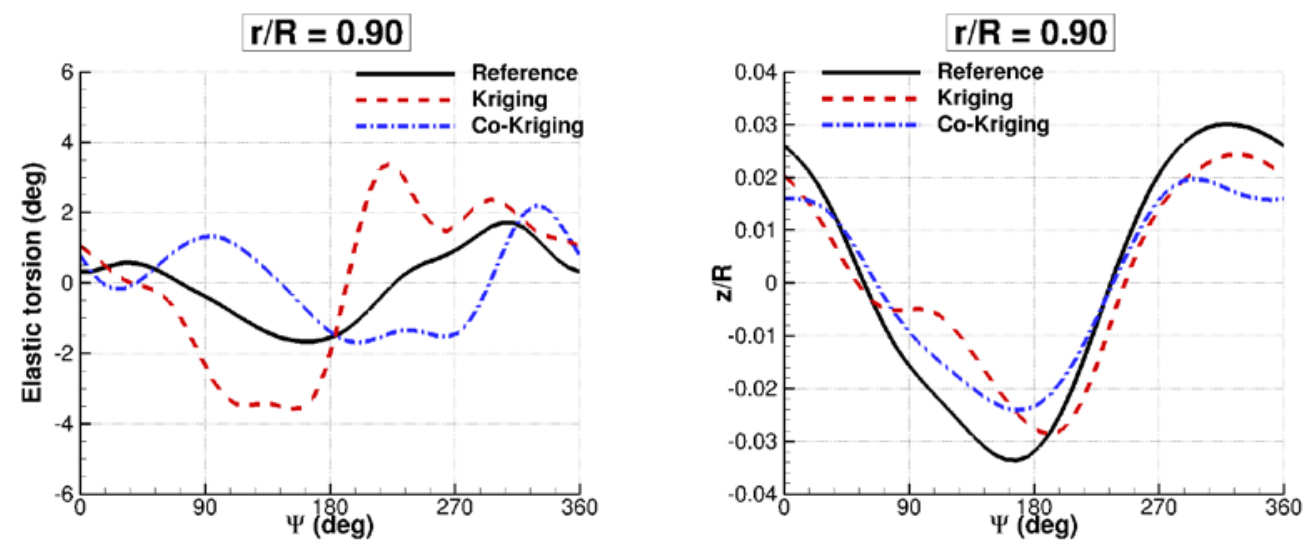

Fig. 18: Elastic torsion and dimensionless flap deformations (mean removed) near the blade tip for the reference, the Kriging and the Co-Kriging optimized blades (HF simulations).

Thanks to its smooth and realistic planform, the torsional deformation of the Co-Kriging optimized blade has a peak-to-peak amplitude reduced by $3^{\circ}$ compared to that of the Kriging optimized blade. The flap evolution of the Co-Kriging optimized blade is also smoother than that of the Kriging optimized blade.

\section{Concluding Remarks}

The optimization of helicopter rotor blades is a challenging problem, as increasingly complex blade planforms are considered. The primary issue is an accurate prediction of the three-dimensional unsteady effects in the optimization loop, necessary for realistic blade planforms and accurate estimation of the power consumed by the main rotor. As the CFD codes are computationally expensive and time-consuming, it is suitable to use surrogate models to replace these high-fidelity simulations.

Two aerodynamic optimization procedures have been presented to define an optimized sweep distribution, by using low and high-fidelity levels of surrogate models, respectively based on Kriging and Co-Kriging methodologies.

The primary findings obtained in this study are:

- The optimization procedure with lifting-line computations replaced by a Kriging model provide an equivalent solution to the optimization procedure based on the coupling between lifting-line and CMA-ES optimizer, but with a factor of 5 reduction in CPU resources.

- A posteriori evaluation of the shaft power of the optimized Kriging rotor with high-level computations CSD/CFD weak coupling predicts a loss of about $1 \%$ in the shaft power with respect to the reference rotor. This indicates that the accuracy of the lifting-line computations and the Kriging models is not sufficient. Inclusion of 
high-fidelity evaluations in the optimization loop is mandatory and can be effective through the use of a Co-Kriging model.

- The blade planform of the optimized rotor resulting from the Co-Kriging optimization procedure is smoother and more realistic than the rotor optimized with a Kriging model. The power benefit is estimated at about $2 \%$ via CFD computations. A limited number of High Fidelity simulations is required to obtain a satisfactory optimized solution. A factor of 6 in CPU time can be estimated with respect to what would have been required the Kriging optimization procedure based on HF simulations.

Further efforts will consist in introducing constraints on the structural loads and stability to ensure realistic optimized blade planforms in terms of aeroelastic behavior. It would also be interesting to validate gradientenhanced Kriging and Co-Kriging models. This will permit the reduction in the number of points in the database, which will lead to a reduction in the CPU time of the optimization procedure. Finally, developing and validating different models to quantify the uncertainty propagation from selected aleatory and/or epistemic variables would provide further insights before performing robust optimization procedures. 


\section{References}

[1] Vanderplaats, G.N., “CONMIN: A Fortran Program for Constrained Function Minimization”, Technical Report, NASA-TMX-62282, August 1973.

[2] Arnaud, G., and Beaumier, P., "Validation of R85/METAR on the Puma RAE Flight Tests", Proceedings of the 18th European Rotorcraft Forum, Avignon, France, 1992.

[3] Zibi, J., Defresne, G., and Costes, M., “A Numerical Procedure for Aerodynamic Optimization of Helicopter Rotor Blades”, Proceedings of the $18^{\text {th }}$ European Rotorcraft Forum, Avignon, France, September 1992.

[4] Dumont, A., Le Pape, A., Peter, J., and Huberson, S., “Aerodynamic Shape Optimization of Hovering Rotors using a Discret Adjoint of the Reynolds-Averaged Navier-Stokes Equations”, Journal of the American Helicopter Society, Volume 56, Number 3, July 2011, pp. 1-11 (11). doi: https://doi.org/10.4050/JAHS.56.032002.

[5] Roca Leon, E., Le Pape, A., Costes, M., Désidéri, J.-A., and Alfano, D., “Concurrent Aerodynamic Optimization of Rotor Blades using a Nash Game Method”, Journal of the American Helicopter Society, Volume 61, Number 2, April 2016, pp. 113 (13). doi: https://doi.org/10.4050/JAHS.61.022009.

[6] Choi, S., Lee, K., Potsdam, M., and Alonso, J.J., "Helicopter Rotor Design using a Time-Spectral and Adjoint-Based Method”, Journal of Aircraft, Volume 51, Number 2, (2014), pp. 412-423. doi: https://doi.org/10.2514/1/C031975.

[7] Mishra, A., Mani, K., Mavripilis, D., and Sitaraman, J., "Helicopter Rotor Design Using Adjoint-Based Optimization in a Coupled CFD-CSD Framework”, Proceedings of the American Helicopter Society $69^{\text {th }}$ Annual Forum, Phoenix, Arizona, USA, May 2013.

[8] Boopathy, K., and Kennedy, G.J. “Adjoint-Based Derivative Evaluation Methods for Flexible Multibody Systems with Rotorcraft Applications”, Proceedings of the 55 ${ }^{\text {th }}$ AIAA Aerospace Sciences Meeting, Grapevine, Texas, 2017. https://doi.org/10.2514/6.2017-1671.

[9] Bailly, J., Ortun, B., Delrieux, Y., and Mercier des Rochettes, H., "Recent Advances in Rotor Aerodynamic Optimization, Including Structural Data Update”, Journal of the American Helicopter Society, Volume 62, Number 2, April 2017, pp. 1-11 (11). doi: htps://doi.org/10.4050/JAHS.62.022009.

[10] Glaz, B., Friedmann, P. P., and Liu, L., "Surrogate Based Optimization of Helicopter Rotor Blades for Vibration Reduction in Forward Flight”, Structural and Multidisciplinary Optimization, Volume 35, Issue 4, April 2008, pp. $341-363$ (23). doi:10.1007/s00158-007-0137-z.

[11] Potsdam, M., Yeo, H., and Johnson, W., "Rotor Airloads Prediction using Loose Aerodynamic/Structural Coupling”, Journal of Aircraft, Volume 43, Number 3, May-June 2006, pp. 732-742. doi:10.2514/1.1006. 
[12] Ortun, B., Potsdam, M., Yeo, H., and Truong, K. V., "Rotor Loads Prediction on the ONERA 7A Rotor using Loose Fluid/Structure Coupling”, Proceedings of the American Helicopter Society $72^{\text {nd }}$ Annual Forum, West Palm Beach, Florida, USA, May 2016.

[13] Jeong, S., Murayama, M., and Yamamoto, K., “Efficient Optimization Design Method Using Kriging Model”, Journal of Aircraft, Volume 42, Number 2, March-April 2005, pp. 413-420 (8). doi:10.2514/1.6386.

[14] Vu, N. A., Lee, J. W., and Shu, J. I., “Aerodynamic Design Optimization of Helicopter Rotor Blades Including Airfoil Shape for Hover Performance”, Chinese Journal of Aeronautics, Volume 26, Issue 1, February 2013, pp. 1-8 (8). doi:10.1016/j.cja.2012.12.008.

[15] Suguira, M., Tanabe, Y., Aoyama, T., Ortun, B., and Bailly, J., “An ONERA/JAXA Co-Operative Research on the Assessment of Aerodynamic Methods for the Optimization of Helicopter Rotor Blades, Phase I”, Proceedings of the $4^{\text {th }}$ Asian/Australian Rotorcraft Forum, Bengaluru, India, November 2015.

[16] Suguira, M., Tanabe, Y., Aoyama, T., Ortun, B., and Bailly, J., “An ONERA/JAXA Co-Operative Research on the Assessment of Aerodynamic Methods for the Optimization of Helicopter Rotor Blades, Phase II”, Proceedings of the $42^{\text {nd }}$ European Rotorcraft Forum, Lille, France, September 2016.

[17] Collins, K. B., Sankar, L. N., and Mavris, D., N., "Application of Low- and High- Fidelity Simulation Tools to Helicopter Rotor Blade Optimization”, Journal of the American Helicopter Society, Volume 58, Number 4, October 2013, pp. 1-10 (10). doi: htps://doi.org/10.4050/JAHS.58.042003.

[18] Wilke, G., "Variable Fidelity Optimization of Required Power of Rotor Blades: Investigation of Aerodynamic Models and Their Application”, Proceedings of the $38^{\text {th }}$ European Rotorcraft Forum, Amsterdam, The Netherlands, September 2012.

[19] Wilke, G., “Multi-Objective Optimizations in Rotor Aerodynamics using Variable Fidelity Simulations”, Proceedings of the $39^{\text {th }}$ European Rotorcraft Forum, Moscow, Russia, September 2013.

[20] Wilke, G., “Applying Multi-Objective Variable-Fidelity Optimization Techniques to Industrial Scale Rotors: Blade Design for CleanSky”, Proceedings of the 41 ${ }^{\text {st }}$ European Rotorcraft Forum, Munich, Germany, September 2015.

[21] Benoit, B., Dequin, A., Kampa, K., von Grünhagen, W., Basset, P.-M., and Gimonet, B. "HOST, A General Helicopter Simulation Tool for Germany and France”, Proceedings of the American Helicopter Society $56^{\text {th }}$ Annual Forum, Virginia Beach, USA, May 2000.

[22] Mc Kay, M. D., Beckman, R. J., and Conover, W. J., "Comparison of Three Methods for Selecting Values of Input Variables in the Analysis of Output from a Computer Code”, Technometrics, Volume 21, Number 2, May 1979, pp. $239-245$. doi: $10.2307 / 1268522$.

[23] Morris, M.D., and Mitchell, T.J., “Exploratory Designs for Computational Experiments”, Journal of Statistical Planning and Inference, Volume 43, Issue 3, February 1995, pp. 381-402. doi:10.1016/0378-3758(94)00035-T 
[24] Krige, D. G., “A Statistical Approach to Some Basic Mine Valuation Problems in the Witwatersrand”, Journal of the Chemical, Metallurgical and Mining Society of South Africa, Volume 52, Number 6, December 1951, pp. 119-139. doi: $10.2307 / 3006914$.

[25] Matheron, G. M., “Principles of Geostatistics”, Society of Economic Geologists, Volume 58, Number 8, December 1962, pp. 1246_1266. doi: https://doi.org/10.2113/gsecongeo.58.8.1246.

[26] Sacks, J., Welch, W. J., Mitchell, T., and Wynn, H.P., “Design and Analysis of Computer Experiments”, Statistical Science, Volume 4, Number 4, November 1989, pp. 409-423.

[27] Kennedy, M., and O’Hagan, "Predicting the Output from a Complex Computer Code when a Cast Approximation is Available”, Biometrika, Volume 87, Number 1, March 2000, pp. 1-13.

[28] Forrester, A., Sobester, A., and Keane, A., "Multi-Fidelity Optimization via Surrogate Modeling”, Proceedings of the Royal Society, Volume 463, Issue 2088, December 2007, pp. 3251-3269. doi: https://doi.org/10.1098/rspa.2007.1900.

[29] Han, Z., Gortz, S., "Hierarchical Kriging Model for Variable-Fidelity Surrogate Modelling”, AIAA Journal, Volume 50, Number 9, September 2012, pp. 1885-1896. doi: https://doi.org/10.2514/1.J051354.

[30] Keane A.J., “Cokriging for Robust Design Optimization”, AIAA Journal, Volume 50, Number 11, November 2012, pp. 2351-2364. doi: https://doi.org/10.2514/1.J051391.

[31] Jamil M., Yang X-S, “A Literature Survey of Benchmark Functions for Global Optimization Problems”, Int. J. Mathematical Modelling and Numerical Optimization, Vol. 4, No. 2, 2013. Doi: 10.1504/IJMMNO.2013.055204.

[32] Arnaud, G., and Beaumier, P., "Validation of R85/METAR on the Puma RAE Flight Tests”, Proceedings of the 18 European Rotorcraft Forum, Avignon, France, September 1992.

[33] Servera, G., Beaumier, P., and Costes, M., “A Weak Coupling Method between the Dynamics Code HOST and the 3D Unsteady Euler Code WAVES”, Proceedings of the $26^{\text {th }}$ European Rotorcraft Forum, The Hague, The Netherlands, September 2000.

[34] Cambier, L., Heib, S., and Plot, S., “The ONERA elsA CFD Software: Input from Research and Feedback from Industry”, Mechanics and Industry, Volume 14, Issue 03, January 2013, pp. 159-174. doi:10.1051/meca/2013056.

[35] Kok, J., "Resolving the Dependance on Freestream Values for the k- Turbulence Model”, AIAA Journal, Vol. 38, No. 7, 2000, pp. 1292-1295. doi:10.2514/2.1101.

[36] Menter, F.R., “Two-Equation Eddy-Viscosity Transport Turbulence Model for Engineering Applications”, AIAA Journal, Vol. 32, No. 8, 1994, pp. 1598-1605. doi:10.2514/3.12149.

[37] Richez, F., and Ortun, B., "Numerical Investigation of the Flow Separation on a Helicopter Rotor in Dynamic Stall Configuration”, Proceedings of the $42^{\text {nd }}$ European Rotorcraft Forum, Lille, France, September 2016. 
[38] Hansen, N., and Kern, S. “Evaluating the CMA Evolution Strategy on Multimodal Test Functions”, Parallel Problem Solving from Nature, PPSN VIII, PPSN 2004, Lecture Notes in Computer Science, Vol. 3242, Springer, Berlin, Germany, 2004. 